\title{
Bioprospección de hongos micorrízicos arbusculares como alternativa para el fortalecimiento del cultivo de aguacate (Persea americana Miller) en Colombia
}

Bioprospecting of fungi mycorrhizal mycorrhizal
as an alternative for the strengthening of the
cultivation of avocado (Persea americana Miller)
in Colombia Bioprospecção fungos micorrízicos arbusculares
como uma alternativa para fortalecer o cultivo
de abacate (Persea americana Miller) na Colômbia Sandra Patricia Montenegro Gómez¹, Silvia Eugenia Barrera Berdugo² \& Carlos Mario Valencia

${ }^{1}$ Licenciada en Biología y Química. Especialista en manejo y conservación de suelos y aguas. Magister en ciencias agrarias, énfasis suelos. Doctora en Ciencias área de concentración microbiología Agrícola. ²Bióloga, Magister y Doctora en Ciencias, énfasis en suelos y nutrición de plantas, área de concentración microbiología del suelo. ${ }^{3}$ Agrónomo, Especialista en Biotecnología Agraria

${ }^{1}$ Centro de Investigación de Agricultura y Biotecnología-CIAB. Dosquebradas. Risaralda. Colombia. ${ }^{2}$ Grupo de Investigación en Ecofisiología Vegetal \& Ecosistemas TerrestresUniversidad Industrial de Santander-UIS. ${ }^{3}$ Gobernación de Antioquia.

1'sandra.montenegro@unad.edu.co, ${ }^{2}$ silviaebarrerab@gmail.com, ${ }^{3}$ carlos.valencia@antioquia.gov.co

\section{Resumen}

El Aguacate (Persea americana Miller) es uno de los frutos de mayor consumo mundial. México es el principal productor y actualmente afronta problemas como el ataque de patógenos a la raíz y recientemente se han registrado impactos en la salud humana por uso de agroquímicos. Una alternativa para afrontar problemáticas en el desarrollo de las plantas ha sido iniciar el correcto manejo de las primeras etapas de producción y con ello mitigar problemas posteriores, en este manejo los microorganismos juegan un papel fundamental. Uno de los métodos es la inoculación con microorganismos del suelo como los hongos micorrízicos arbusculares (HMA); diversos estudios han demostrado que su colonización radicular en las plantas contribuye con la absorción de agua y nutrientes; lo cual indica que su potencialización puede contribuir en el manejo sustentable de los cultivos y como opción para fortalecer la producción. Colombia ocupa el quinto lugar en productividad a nivel mundial y lo que más resta competitividad local es el costo de los agroinsumos; en este sentido se podrían fortalecer investigaciones encaminadas a la bioprospección de HMA específicos para diversas variedades de aguacate en las regiones colombianas, ya que son poco los avances en esta temática. Avanzar tecnológicamente en el 
manejo de cultivos y generar estrategias sostenibles hace parte de las perspectivas y retos de la cadena del aguacate en Colombia. Adicionalmente es prioritario entrar en la dinámica de la demanda mundial con preferencia de alimentos más sanos y cuya producción sea amigable con el medio ambiente.

Palabras clave: Hongos micorrízicos arbusculares, Aguacate (Persea americana, Miller). Bioprospección, sustentabilidad.

\section{Abstract}

Avocado (Persea americana Miller) is one of the most consumed fruits in the world. Mexico is the main producer of avocado and currently Mexico faces problems such as the attack of pathogens to the root and recently there have been impacts to human health by the use of agrochemicals. An alternative to deal with problems in the development of plants has been to start the correct management of the first stages of production and with that to mitigate later problems, in this management microorganisms play a fundamental role. The inoculation with arbuscular mycorrhizal fungi (AMF) is an important method. Several studies have shown that the root colonization in plants help to the absorption of water and nutrients. It indicates that the AMF can contribute to the sustainable management of crops as an option to strengthen production. Although Colombia is the top 5 of productivity in the world level, local competitiveness is the cost of agro-inputs. To the other hand, the research aiming to bioprospection of AMF specific for avocado varieties in Colombian regions, is necessary and could be strengthened this field because of it is little know currently about advances in this theme and its influence on the strengthening of Colombian agriculture. Advancing in the crop management and generating sustainable strategies are the perspectives and challenges for the chain of avocado in Colombia. Indeed, it is a priority to get in at the dynamics of global demands preferring healthier food and friendly production to the environment.
Key-words: Arbuscular mycorrhizal fungi, Avocado (Persea americana, Miller). Bioprospecting, sustainability.

\section{Resumo}

Abacate (Persea americana, Miller) é uma das frutas mais consumidas no mundo. México é o maior produtor e atualmente passa por problemas como o ataque de patógenos na raiz e recentemente tem-se encontrado que afeta a saúde humana pelo uso de agroquímicos. Uma alternativa para resolver os problemas no desenvolvimento das plantas foi iniciar com o tratamento correto nos estágios iniciais de produção e, assim, mitigar problemas posteriores. É aqui que os microorganismos desempenham um papel fundamental. Um método é a inoculação com microrganismos do solo como os fungos micorrízicos arbusculares (FMA). Estudos têm mostrado que a colonização da raiz contribui na absorção de água e nutrientes; indicando que potenciar seu uso pode contribuir para uma gestão agrícola sustentável e assim fortalecer a produção. Por outro lado, Colômbia ocupa o quinto lugar em produtividade no mundo e que mais resta na competitividade local é o custo dos insumos agrícolas; com relação a isto, deve-se fortalecer a pesquisa de bioprospecção em FMA específicos para diversas variedades de abacate nas regiões colombianas, porque na atualidade, sabe-se pouco sobre os avanços neste tema e sua influência sobre o fortalecimento da agricultura colombiana. Avançar tecnologicamente em manejo de culturas e gerar estratégias sustentáveis é parte das perspectivas e desafios da cadeia Colombiana do abacate. Além disso, é uma prioridade para a dinâmica da demanda global de alimentos de preferência mais saudáveis visando uma produção ambientalmente amigável.

Palavras-chave: Fungos micorrízicos arbusculares, Abacate (Persea americana, Miller). Bioprospecção, sustentabilidade. 


\section{Introducción}

El aguacate (Persea americana, Miller) es un fruto de gran consumo mundial, con un comercio dinámico y en crecimiento. Colombia se ubica entre los cinco primeros lugares de producción de acuerdo a datos registrados por la FAO STAT hasta el año 2014 , con producción anual de 288.739 t. México ocupa el primer lugar con 1.520 .695 t, este país también ocupa el primer lugar mundial en consumo per capita y exportación (Bárcenas et al., 2011). Pese a su privilegiada ubicación, México afronta problemas de producción, como el ataque de patógenos a la raíz (Graham, 2001; Morales-García, 2009, Contreras, 2012) e impactos sobre la salud humana por uso de agroquímicos (El financiero, 2016). Una alternativa para afrontar algunas de las problemáticas ha sido iniciar el correcto manejo de las primeras etapas de producción y con ello mitigar problemas posteriores, en este manejo los microorganismos juegan un papel fundamental. Uno de los métodos es la inoculación con microorganismos del suelo, como los hongos micorriza arbuscular HMA, (Contreras, 2012). Diversos estudios han demostrado que HMA además de favorecer la absorción y aprovechamiento de los nutrimentos del suelo en estas plantas, incrementan su tasa fotosintética y promueven aumento del crecimiento y mejora en la salud de las mismas. (Menge et al., 1980; AzcónAguilar et al., 1992; Bárcenas et al., 2011). El papel de los HMA junto a microorganismos solubilizadores de fosfato ha dado buenos resultados en plantaciones de aguacate en otros países como Chile, Brasil y Estados Unidos (Thies \& Grossman, 2006).

Factores como la especie vegetal y el tipo de suelo pueden influir en el establecimiento de la asociación HMA vs planta, así como otros factores bióticos o abióticos, por lo tanto investigaciones al respecto, deben direccionarse hacia poblaciones de HMA nativas, ya que puede existir adaptación y/o restricciones por factores locales que en algún momento podrían transformar esta relación comúnmente mutualista en una relación parasitaria (Lasso-Rivas, 2015). Tanto poblaciones nativas como foráneas de hongos micorrízicos arbusculares han sido usadas en la agricultura como inoculantes biológicos. Ejemplos donde se evalúa el efecto de inóculos comerciales en diferentes cultivos son abundantes (Motta \& Munévar, 2005; Douds Jr et al., 2007; Castillo et al., 2009; Fernandez et al., 2011), observándose un efecto positivo en la planta derivado de la interacción con el hongo, y aunque suelo con esporas y propágulos es usado como inóculo, otros productos como inóculos líquidos, usados principalmente en horticultura, actúan como inoculante de semillas o son aplicados alrededor del tallo (http://mycorrhizae.com/mycoapply-products/liquid/mycoapplyliquid-endo/).

\section{Importancia de la interacción HMA y plantas de Aguacate}

Los hongos micorrízicos desarrollan una extensa red de hifas en el suelo y ofrecen una eficiente transferencia horizontal de agua y nutrientes para las plantas a partir de áreas especializadas Ilamadas interfaces simbióticas que interactúan con la planta hospedera (Bonfante \& Genre, 2010). Los mecanismos de interacción planta/hongo micorrízico están asociados a la especie de planta hospedera y a la estructura y función de los hongos, estos pueden ser ectomicorrizas ECM, micorrizas de orquídeas, ericoides y micorriza arbusculares MA (van der Heijden et al., 2015). En la raíz las ECM forman un manto alrededor de la raíz y penetran de forma intercelular la corteza de la misma, se ha encontrado que colonizan un número menor de plantas en comparación con las MA, los cuales colonizan de forma inter e intracelular la corteza de la raíz, formando arbúsculos, dentro de la célula vegetal (Figura 1). Los HMA interactúan simbióticamente con el $80 \%$ de las familias de plantas terrestres encontrándose en casi todos los ecosistemas terrestres (Smith \& Read, 2008; Carreón-Abud et al., 2013), generando una fuerte influencia sobre el crecimiento y la productividad vegetal (van der Heijden et al., 2015). Por su parte la asociación simbiótica con la mayoría de las plantas de cultivo ha despertado gran interés en contextos agronómicos para el uso potencial de HMA en la producción sostenible con tendencia a la disminución en la aplicación de insumos químicos (Lanfranco et al., 2016). 


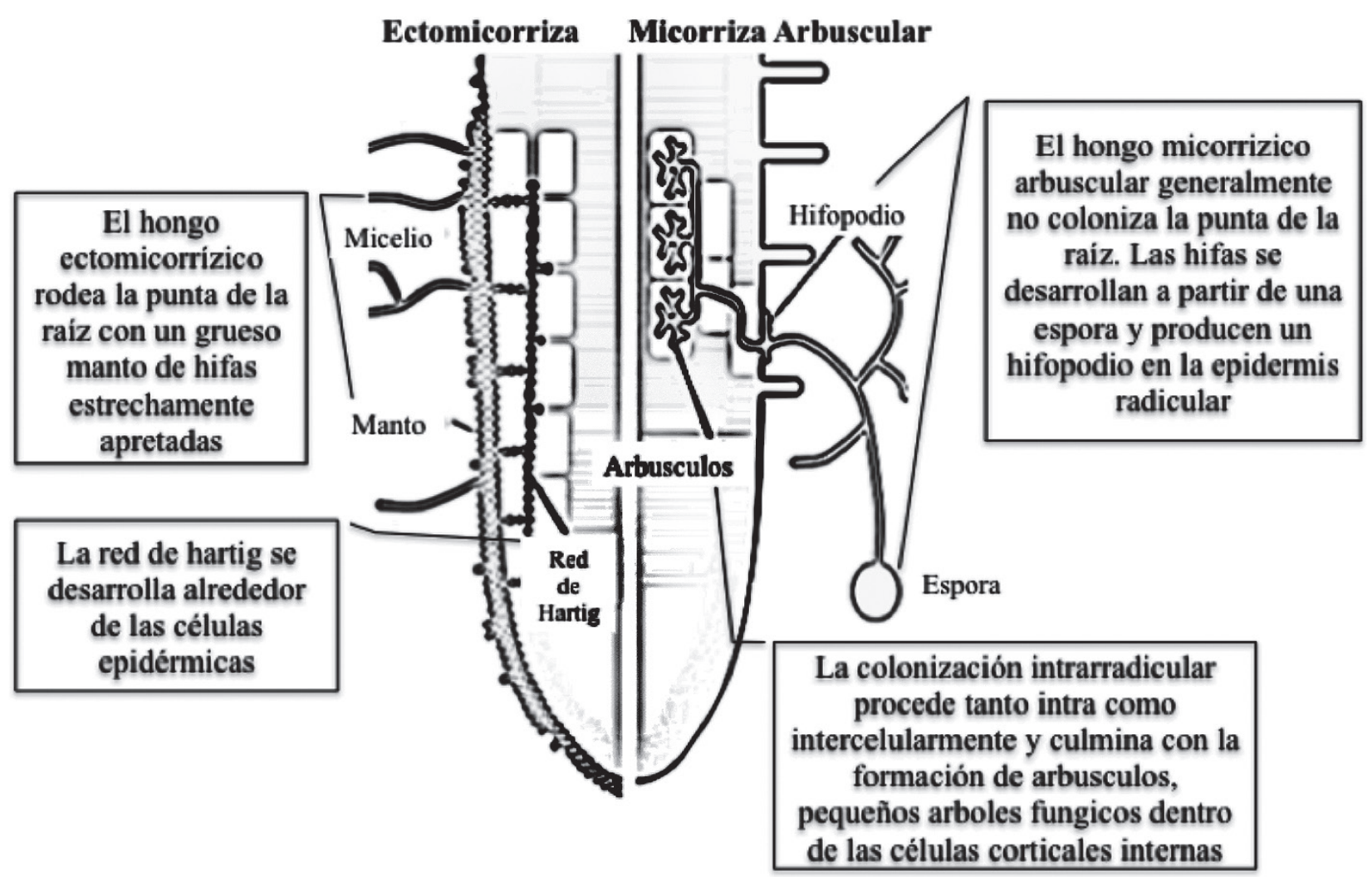

Figura 1. Ilustración de las estructuras de colonización de raíces en interacciones ectomicorrízicas (izquierda) y micorrízicas arbusculares (derecha). Fuente: Bonfante \& Genre (2010).

Entre otros cultivos de interés agrícola, diversas leguminosas, cítricos, papaya, aguacate, manzana, mango, fresa y durazno han sido reportados por su interacción y resultados benéficos en desarrollo y productividad asociados a la presencia de HMA. En lo que respecta el aguacate; esta es una planta con pocos pelos absorbentes y la absorción de agua y nutrientes la realiza a través de tejidos primarios de las puntas de las raíces (Godínez et al., 2000; Lavaire \& Morazán, 2013), por lo tanto la asociación microbiológica aguacate/hongos micorrízicos, se convierte en un mecanismo que facilita la exploración del suelo por parte de esta planta de raíz pivotante y muy ramificada en los primeros $60 \mathrm{~cm}$. (Whiley et al., 1988a). Plantas con sistemas radiculares abundantes pero con pocos pelos absorbentes, como yuca, cebolla y cítricos, pueden ser altamente dependientes de la micorrización (Sánchez, 1999), mientras que plantas con un sistema radicular con abundantes raíces finas y pelos radiculares largos pueden ser poco dependientes de esta (Sieverding, 1991).
Desde la caracterización de un transportador de fosfato de alta afinidad, TP, en un HMA por Harrison \& van Buuren (1995), la asociación simbiótica entre plantas y estos hongos ha sido objeto de estudio para el fortalecimiento en la toma de nutrientes en las planta y una alternativa para la fertilización agrícola sustentable, la cual complementada con otras prácticas de fertilización orgánica ha demostrado resultados muy favorables en transporte de $\mathrm{P}$ al interior de las plantas (Velez \& Sánchez, 2014). Según lo reportado por Allen \& Shachar-Hill (2009), Sieh et al. (2013) y Berruti (2015), el fosforo inorgánico -Pi-, N y S se pueden transferir a las plantas a través de HMA. Actualmente se sabe que existe asimilación de $\mathrm{K}^{+}$derivado de la simbiosis HMA-planta, la cual podría estar relacionada con la tolerancia de la planta al estrés abiótico (Garcia \& Zimmermann, 2014; Berruti, 2015). Recientemente, se han publicado estudios de metanálisis, centrados en la respuesta de cultivos a la contribución de diferentes 
concentraciones de micronutrientes provenientes de la interacción con HMA (Lehmann et al., 2014; Lehmann \& Rillig, 2015; Berruti, 2015). Los resultados muestran incrementos de $\mathrm{Zn}$ en tejidos de varias plantas de interés agrícola en diferentes ambientes (Lehmann et al., 2014), un efecto positivo en la absorción de Cu (Lehmann \& Rillig, 2015) y mejoramiento en la productividad y en el contenido nutricional de $\mathrm{Fe}$ y $\mathrm{Zn}$ en garbanzo $\mathrm{Ci}$ cer arietinum (Pellegrino \& Bedini, 2014).
De acuerdo a los planes de fertilización, de modo general la extracción de nutrientes en la fruta fresca del aguacate, en orden descendente sería la siguiente: Potasio $\left(\mathrm{K}_{2} \mathrm{O}\right)>$ Nitrógeno(N)>Fósforo $\left(\mathrm{P}_{2} \mathrm{O}_{5}\right)>$ Calc io(CaO)>Magnesio(MgO)>Azufre(S) (Bernal \& Díaz, 2005). Algunos estudios revelan la efectividad de la asociación de la planta con HMAs. En la Tabla 1, se detallan algunas experiencias internacionales relevantes a nivel latinoamericano, donde México, como mayor productor del mundo lidera estudios basados en asociación de aguacate con HMA.

Tabla 1. Algunas experiencias internacionales relevantes a nivel latinoamericano en producción de aguacate asociado a HMA

\begin{tabular}{|c|c|c|}
\hline Publicación & Autor/año & País \\
\hline $\begin{array}{l}\text { Diversidad de hongos micorrícicos arbusculares provenientes } \\
\text { de la rizósfera de aguacate (Persea americana Miller) y selección } \\
\text { de plantas trampa para su propagación }\end{array}$ & $\begin{array}{l}\text { Carreón et al. } \\
(2016)\end{array}$ & México \\
\hline $\begin{array}{l}\text { Interacción de inoculantes micorrízicos arbusculares y estiércol } \\
\text { de pollo en la producción de portainjertos de aguacate }\end{array}$ & $\begin{array}{l}\text { Carreón et al. } \\
(2015)\end{array}$ & México \\
\hline $\begin{array}{l}\text { Inoculación micorrízico arbuscular en portainjertos de plantas } \\
\text { de aguacate cv 'Hass' en viveros de Michoacán, México }\end{array}$ & $\begin{array}{l}\text { Carreón et al. } \\
\text { (2014) }\end{array}$ & México \\
\hline $\begin{array}{l}\text { Aislamiento y propagación de cultivos puros de hongos micorrízicos } \\
\text { arbusculares provenientes de huertas de aguacate con diferente } \\
\text { manejo agrícola por la técnica de minirizotrón. }\end{array}$ & $\begin{array}{l}\text { Carreón-Abud et al. } \\
\text { (2013) }\end{array}$ & México \\
\hline $\begin{array}{l}\text { Etapas óptimas de inoculación micorrízica en plántulas de aguacate } \\
\text { (Persea americana) }\end{array}$ & Contreras, (2012) & México \\
\hline $\begin{array}{l}\text { Utilización de cepas eficientes de hongos micorrízicos } \\
\text { arbusculares en el desarrollo de portainjertos de aguacate } \\
\text { en un sustrato suelo-cachaza }\end{array}$ & Rivera et al. (2011) & Cuba \\
\hline $\begin{array}{l}\text { Catálogo de hongos micorrizógenos arbusculares de huertos } \\
\text { de aguacate (Persea americana, Miller) de Michoacán. }\end{array}$ & $\begin{array}{l}\text { Bárcenas et al. } \\
\text { (2011) }\end{array}$ & México \\
\hline $\begin{array}{l}\text { Elementos minerales y carbohidratos en plantones de aguacate } \\
\text { (Persea americana Miller) 'carmen' inoculados } \\
\text { con micorrizas arbusculares. }\end{array}$ & $\begin{array}{l}\text { Da Silveira et al. } \\
(2003)\end{array}$ & Brasil \\
\hline $\begin{array}{l}\text { Aplicación de vermicomposta y hongos micorrízicos en la producción } \\
\text { de planta de aguacate en vivero. }\end{array}$ & Reyes et al. (2001) & México \\
\hline $\begin{array}{l}\text { Simbiosis Micorrízica y vermicomposta en el Desarrollo de Porta } \\
\text { injertos de Aguacate crecidos en Sustratos Agrícola y Forestal. }\end{array}$ & Reyes et al. (2000) & México \\
\hline $\begin{array}{l}\text { Micorrizas en la agricultura: contexto mundial e investigación } \\
\text { realizada en costa rica. }\end{array}$ & $\begin{array}{l}\text { Blanco, et al. } \\
\text { (1997). }\end{array}$ & Costa Rica \\
\hline
\end{tabular}




\section{HMA y prospección productiva de aguacate en Colombia}

El aguacate es una planta que se puede adaptar a diferentes condiciones climáticas. La producción de aguacate en Colombia pasó de tener una balanza comercial deficitaria en 2010 a ser superavitaria a partir del 2013. Los costos de producción también se han ido incrementado principalmente por el valor de los agroinsumos, que en síntesis es el componente que más resta competitividad en la fase productiva para la industria local (Minagricultura, 2015). Avanzar tecnológicamente en el manejo de cultivos y generar estrategias sostenibles, hace parte de las perspectivas y retos de la cadena del aguacate en Colombia de acuerdo a Barreño (2014), en este sentido se podrían fortalecer investigaciones encaminadas a la bioprospección de HMA específicos para diversas variedades de aguacate en las regiones colombianas ya que hasta ahora es poco lo que se ha trabajado en esta temática. En la Tabla 2 se presentan algunos estudios relevantes realizados en el país los cuales se basan principalmente en porcentaje de colonización, caracterización de esporas nativas y respuesta de la planta.

Tabla 2. Algunas experiencias colombianas en producción de Aguacate asociado a HMA

\begin{tabular}{|c|c|c|}
\hline Ubicación & Autor/año & Región \\
\hline $\begin{array}{l}\text { Caracterización molecular, análisis morfológico y colonización micorrízica } \\
\text { en la rizósfera del aguacate (Persea americana, Mill) en Caldas, Colombia }\end{array}$ & Rivera et al. (2016) & Caldas \\
\hline $\begin{array}{l}\text { Caracterización y obtención de cultivos puros de esporas nativas } \\
\text { del genero Glomus sp. Asociadas a rizósfera de aguacate } \\
\text { (Persea Americana, Mill) }\end{array}$ & $\begin{array}{l}\text { Aranguren et } \\
\text { al.(2015) }\end{array}$ & Tolima \\
\hline $\begin{array}{l}\text { Las interacciones de la rizósfera como base para el manejo eficiente } \\
\text { de la nutrición y sanidad del aguacate en Colombia }\end{array}$ & $\begin{array}{l}\text { Bolaños et al., } \\
\text { 2008-2011. Presente } \\
\text { publicación (2014) }\end{array}$ & $\begin{array}{l}\text { Tolima y Valle } \\
\text { del Cauca }\end{array}$ \\
\hline $\begin{array}{l}\text { Respuesta de la inoculación de micorrizas en plántulas de aguacate } \\
\text { (Persea americana, Miller) variedad "Hass" en diferentes sustratos. }\end{array}$ & Melo (2011) & $\begin{array}{l}\text { Valle } \\
\text { del Cauca }\end{array}$ \\
\hline $\begin{array}{l}\text { Efecto de la inoculación conjunta con hongos micorrizales y microorganis- } \\
\text { mos solubilizadores de fósforo en plantas de aguacate }\end{array}$ & Serna (2013) & Antioquia \\
\hline $\begin{array}{l}\text { Efecto de la micorrización en plantas de aguacate (Persea americana } \\
\text { Mill.) variedad Hass y Nativo Antillano durante la fase de vivero. }\end{array}$ & Pérez (2013) & Caldas \\
\hline $\begin{array}{l}\text { Use of soil microorganisms as a biotechnological strategy to enhance } \\
\text { avocado (persea americana)-plant phosphate uptake and growth }\end{array}$ & Osorio et al. (2012) & Antioquia \\
\hline $\begin{array}{l}\text { Colonización micorrícica en plantas de aguacate (Persea americana L.) } \\
\text { en fase de vivero }\end{array}$ & $\begin{array}{l}\text { Montañez et al. } \\
(2010)\end{array}$ & Meta \\
\hline $\begin{array}{l}\text { Efecto de la micorrización en plantas de aguacate (Persea americana L.) } \\
\text { durante la fase de vivero en suelos provenientes de los Llanos Orientales }\end{array}$ & $\begin{array}{l}\text { Montañez et al. } \\
(2009)\end{array}$ & Meta \\
\hline
\end{tabular}

El consumo global en el mercado agrícola presenta nuevas tendencias enmarcadas en agricultura sostenible, direccionadas a mejorar la calidad, el rendimiento y reducir al mínimo el uso de prácticas agrícolas tradicionales que consumen energía y contaminan el medio ambiente (Montenegro \& Hernandez, 2015). En el año 2002 se publicó el libro: "Aproximación al estado actual de la bioprospección" con el resultado de diversos trabajos de investigación orientados hacia el estudio de las micorrizas (Melgarejo et al., 2002). A pesar de esta iniciativa, el avance no ha sido relevante en el fortalecimiento de la agricultura colombiana. En lo que respecta al aguacate y su crecimiento productivo, es prioritario entrar en la dinámica de la demanda mundial con preferencia de alimentos más sanos y cuya producción sea 
amigable con el medio ambiente, sin descuidar las condiciones de vida de los trabajadores rurales y la de sus familias (Fonseca, Muñoz \& Cleves, 2011), por lo tanto las razones para iniciar prácticas que favorezcan el uso sustentable del suelo, incluyendo la relación simbiótica planta-HMA, se hacen cada vez más necesarias ya que el aumento de productividad del Aguacate en Colombia basada en prácticas agrícolas tradicionales, podría desencadenar efectos negativos tanto en plantas como en cultivadores como ocurre actualmente en México y su desbordada producción de Aguacate (El financiero, 2016). Una proyección sustentable podría basarse en la implementación productiva de aguacate basada en servicios ecosistémicos de regulación, sustentada en el mantenimiento del suelo como parte de uno de estos servicios proporcionado por los ecosistemas, que comúnmente son visibles solamente cuando los daños implican perdidas difíciles de recuperar (FAO, 2017; Corredor, Fonseca \& Páez., 2012).

\section{Conclusiones}

En Colombia, se ha estudiado poco sobre la producción de aguacate y su asociación simbiótica con HMA como herramienta biotecnológica de biobrospección. El cultivo de aguacate en Colombia indica una proyección comercial en alza al igual que el costo de los insumos agrícolas, lo cual resta competitividad en la fase productiva para la industria local y alternativas de mejoramiento en sistema de nutrición a partir de la potencialización de HMA, que podría contribuir hacia el cumplimiento de uno de los retos y perspectivas de la cadena de aguacate en Colombia fundamentada en la generación de estrategias sostenibles.

\section{Literatura citada}

1. Allen, J. W. \& Shachar-Hill, Y. (2009). Sulfur transfer through an arbuscular mycorrhiza. Plant Physiol. 149, 549-560. doi: 10.1104/pp.108. 129866

2. Aranguren, Aroca, R., A.; Ocampo, Guerrero M. L. \& Mesa López, N. (2015). Caracterización y obtención de cultivos puros de esporas nativas del género Glomus $s p$. Asociadas a rizosfera de aguacate (Persea Americana Mill). Revista de la Asociación Colombiana de Ciencias Biológicas. 25, p.50-60.
3. Azcón-Aguilar, C, Barceló, A, Vidal, M. \& De la Viña, G. (1992) Further studies on the influence of mycorrhizae on growth and development of micropopagated avocado plants. Agronomie. 12:837- 840.

4. Bárcenas Ortega, A., Varela-Fregoso, L. Stürmer, S.L. \& Chávez-Bárcenas, AT. (2011). Catálogo de hongos micorrizógenos arbusculares de huertos de aguacate (Persea americana Miller) de Michoacán, México. Universidad Michoacana de San Nicolás de Hidalgo. Congreso mundial del aguacate. Recuperado de http:// www.congresomundialdelaguacate2011.com/userfiles/ file/Ana\%20Barcenas-Ortega\%201130-1150.pdf

5. Barreño F. (2014). Estado actual y perspectivas de la cadena del aguacate en Colombia. Ministerio de Agricultura y Desarrollo rural, Republica de Colombia.

6. Bernal E., J.A. \& Diaz D., C.A. (2005). Tecnología para el Cultivo del Aguacate. Corporación Colombiana de Investigación Agropecuaria, CORPOICA, Centro de Investigación La Selva, Rionegro, Antioquia, Colombia. Manual Técnico 5. 241 p.

7. Blanco, F. A. \& Salas, E. A. (1997). Micorrizas en la agricultura: contexto mundial e investigación realizada en costa rica. Agronomía Costarricense 21(1): 55-67.

8. Berruti, A., Lumini, E., Balestrini, R. \& Bianciotto, V. (2015). Arbuscular Mycorrhizal Fungi as Natural Biofertilizers: Let's Benefit from Past Successes. Frontiers in Microbiology, 6, 1559. http://doi.org/10.3389/ fmicb.2015.01559

9. Bolaños Benavides, M. M. (2014). Manejo de Suelos en la producción de Alimentos Ecológicos -Las interacciones de la rizósfera como base para el manejo eficiente de la nutrición y sanidad del aguacate en Colombia. C. I. Tibaitatá de Corpoica. VI Congreso Nacional Hortofrutícola. Recuperado de: http://www.asohofrucol.com. co/archivos/biblioteca/4Manejo\%2

10. Bonfante, P. \& Genre, A. (2010) Mechanisms underlying beneficial plant- fungus interactions in mycorrhizal symbiosis. Nature Communications 1, 48. doi: 10.1038/ncomms 1046 .

11. Carreón-Abud, Y., Jerónimo-Treviño, E., Beltrán-Nambo, M. A., Martínez-Trujillo, M., Trejo Aguilar, D. \& Gavito, M. E. (2013). Aislamiento y propagación de cultivos puros de hongos micorrízicos arbusculares provenientes de huertas de aguacate con diferente manejo agrícola por la técnica de minirizotrón. Revista mexicana de micología, 37, 29-39. Recuperado de http://www. scielo.org.mx/scielo.php?script=sci_arttext\&pid=S018731802013000100005\&lng=es\&tlng=es.

12. Carreón Abud, Y., Aguirre Paleo, S., Gavito, M.E., Mendoza Solís, D.J., Juárez Chávez, R., Miguel Martínez TrujiIlo, M. \& Trejo Aguilar, D. (2014). Inoculación micorrízico arbuscular en porta injertos de plantas de aguacate (Persea americana Miller) cv 'Hass' en viveros de Michoacán, México. Revista Mexicana de Ciencias Agrícolas Vol.5 Núm.5 30 de junio 13 de agosto, p. 847-857. Recuperado de: http://www.scielo.org.mx/scielo.php?script=sci_arttex t\&pid=S2007-09342014000500010

13. Carreón-Abud, Y., Vega-Fraga, M. \& Gavito, M.E. (2015). Interacción de inoculantes micorrízicos ar- 
busculares y estiércol de pollo en la producción de portainjertos de aguacate. Journal of soil science and plant nutrition, vol.15 no.4, 867-881. Versión On Line ISSN 0718-9516. Recuperado de: http://www.scielo.cl/scielo.php?script=sci_arttex t\&pid=S0718-95162015000400005 http://dx.doi. org/10.4067/S0718-95162015005000060

14. Carreón-Abud Y., Gómez Dorantes, N., Beltrán Nambo M. A, Alvarado-Herrejón M. \& Varela-Fregoso L. (2016). Diversidad de hongos micorricícos arbusculares provenientes de la rizósfera de aguacate (Persea americana Mill) y selección de plantas trampa para su propagación. Revista Biológicas Vol. 18, No. 2, 1-9. Recuperado de: https://www.biologicas.umich. $\mathrm{mx} /$ index.php/biologicas/article/view/BiologicasVol18No2_2106_1-9/205

15. Castillo, Claudia., Sotomayor, L., Ortiz, C., Leonelli, C., Borie, F. \& Rubio, R. (2009). Effect of arbuscular mycorrhizal fungi on an ecological crop of chili peppers (capsicum annuum I.). Chileanjar, 69(1), 79-87.

16. Contreras Bernal, M. M. (2012). Etapas óptimas de inoculación micorrízica en plántulas de aguacate (Persea americana). Universidad veracruzana. Recuperado de: http://cdigital.uv.mx/bitstream/123456789/31358/1/ manuelmiguelcontrerasbernal.pdf

17. Corredor Camargo, E., Fonseca Carreño, J. \& Páez Barón, E. (2012). Los servicios ecosistémicos de regulación: tendencias e impacto en el bienestar humano. Revista de Investigación Agraria y Ambiental, 3(1), enero-junio, 77-83. Recuperado de: http://hemeroteca. unad.edu.co/index.php/riaa/article/view/936/934

18. Da Silveira, S.V., De Souza, P.V.D., Koller, O.C. \& Schwarz, S.F. (2003). Elementos minerales y carbohidratos en plantones de aguacate (Persea americana Miller) 'carmen' inoculados con micorrizas arbusculares. Departamento de Horticultura e Silvicultura. Universidade Federal do Rio Grande do Sul. Actas V Congreso Mundial del Aguacate, p. 415-420. Recuperado de http://www. avocadosource.com/WAC5/Papers/WAC5_p415.pdf.

19. Douds D.D., Jr, Nagahashi G., Reider C. \& Hepperly P.R. (2007). Inoculation with arbuscular mycorrhizal fungi increases the yield of potatoes in a high $P$ soil. Biol Agric Hortic, 25, 67-78.

20. El financiero (2016). Economía: La 'fiebre verde' del aguacate en México, un negocio tóxico. Recuperado de: http://www.elfinanciero.com.mx/economia/la-fiebreverde-del-aguacate-en-mexico-un-negocio-toxico.html

21. FAO (2017). Servicios ecosistémicos y biodiversidad. Recuperado de: http://www.fao.org/ecosystem-services-biodiversity/background/regulatingservices/es/.

22. FAO STAT (2014). Datos. Producción-Cultivos. Mundo Lista. Producto Aguacate. Recuperado de : http://www. fao.org/faostat/es/\#data/QC

23. Fernández, F.; Dell'Amico, J.M., Angoa, M.V. \& de la Providencia, E. (2011). Use of a liquid inoculum of the arbuscular mycorrhizal fungi Glomus hoi in rice plants cultivated in a saline Gleysol: A new alternative to inoculate Journal of Plant Breeding and Crop Science, 3(2), 24-33. Available online http://www.academicjournals.org/jpbcs
24. Fonseca C., J., Muñoz P., N. \& Cleves L., J. (2011). El sistema de gestión de calidad: elemento para la competitividad y la sostenibilidad de la producción agropecuaria colombiana. Revista De Investigación Agraria y Ambiental, 2(1), enero-junio, 9-22. Recuperado de: http://hemeroteca.unad.edu.co/index.php/riaa/article/ view/909/903

25. Garcia, K. \& Zimmermann, S. D. (2014). The role of mycorrhizal associations in plant potassium nutrition. Front. Plant Sci. 5:337. doi: 10.3389/fpls.2014.00337

26. Godínez, M., Martínez, M., Melgar, N. \& Méndez, W. (2000). El cultivo del aguacate en Guatemala. PROFRUTA, MAGA, Guatemala, Guatemala. 35 p.

27. Graham, J. H. (2001). What Do Root Pathogens See in Mycorrhizas? New Phytologist 149: 357-359.

28. Harrison, M. J. \& van Buuren, M. L. (1995). A phosphate transporter from the mycorrhizal fungus Glomus versiforme. Nature 378, 626-629. doi: 10.1038/378626a0.

29. Lanfranco L, Bonfante P. \& Genre, A. (2016). The Mutualistic Interaction between Plants and Arbuscular Mycorrhizal Fungi. Microbiol Spectrum 4(6): FUNK-0012-2016. doi:10.1128/microbiolspec.FUNK-0012-2016.

30. Lasso-Rivas, N. (2015). Efecto de la radiación ultravioleta y la colonización micorrízica en árboles pioneros de la región oriental de América del Norte. Revista de Investigación Agraria y Ambiental, 6(1), enero-junio, 53 - 66. Recuperado de: http://hemeroteca.unad.edu. co/index.php/riaa/article/view/1263/1599

31. Lavaire, L. \& Morazán, F. (2013). Manual técnico del cultivo de aguacate en honduras (Persea americana Mill). Programa Nacional de Desarrollo Agroalimentario de la Secretaría de Agricultura y Ganadería (SAG). Honduras. 58p.

32. Lehmann, A. \& Rillig, M. C. (2015). Arbuscular mycorrhizal contribution to copper, manganese and iron nutrient concentrations in crops-A meta- analysis. Soil Biol. Biochem. 81, 147-158. doi: 10.1016/j.soilbio.2014.11.013

33. Lehmann, A., Veresoglou, S. D., Leifheit, E. F. \& Rillig, M. C. (2014). Arbuscular mycorrhizal influence on zinc nutrition in crop plants-A meta-analysis. Soil

34. Menge, A, LaRue, J, Labanauskas, K \& Johnson, L. (1980). The effect of two mycorrhizal fungi upon growth and nutrition of avocado seedlings grown with six fertilizer treatments, J. Amer. Soc. Hort. Sci. 105:400-404.

35. Melgarejo, L. M., J. Sánchez, A. Chaparro, F. NEWMARK, M. Santos-Acevedo, C. Burbano \& Reyes, C. (2002). Aproximación al estado actual de la bioprospección en Colombia Bogotá: Cargraphics 334p.--(Serie de Documentos Generales INVEMAR No.10). Recuperado de: http://www.invemar.org. co/redcostera1/invemar/docs/ESTADO_BIOPROSPECCION.pdf

36. Minagricultura (2015). Organización de cadena productiva del aguacate. Recuperado de: https://sioc. minagricultura.gov.co/Aguacate/Documentos/002\%20 -\%20Cifras $\% 20$ Sectoriales/002\%20-\%20Cifras $\% 20$ Sectoriales\%20-\%202015\%20Diciembre.pdf 
37. Montenegro Gómez, S. \& Hernández Ossa, Y. (2015). Biotecnología aplicada al desarrollo agropecuario colombiano. Revista de Investigación Agraria y Ambiental, 6(2), julio-diciembre, 97 - 108. Recuperado de: http://hemeroteca.unad.edu.co/index.php/riaa/article/ view/1408/1732

38. Morales-García, J.L. 2009. Enfermedades de importancia económica en el cultivo de aguacate en Michoacán, México. III Congreso latinoamericano del aguacate, del 10-12 de noviembre, Medellín, Colombia. pp. 15-31.

39. Motta D., Munévar, F. (2005). Respuesta de Plántulas de Palma de Aceite a la Micorrización. Palmas, 26 (3): 11-20.

40. Osorio Vega, Nelson Walter, Serna Gómez, Sandra Luz \& Montoya Restrepo, Beatriz Elena. (2012). Use of soil microorganisms as a biotechnological strategy to enhance avocado (persea americana)-plant phosphate uptake and growth. Revista Facultad Nacional de Agronomía, Medellín, 65(2), 6645-6657. Recuperado de. http://www. scielo.org.co/scielo.php?script=sci_arttext\&pid=S030428472012000200009\&lng=en\&tlng=.

41. Montañez Orozco, B.I. (2009). Efecto de la micorrización en plantas de aguacate (Persea americana Lineo.) durante la fase de vivero en suelos provenientes de los Llanos Orientales. Universidad Nacional de Colombia Facultad de Ciencias, Instituto de Ciencias Naturales y Exactas Bogotá, Colombia. Recuperado de: https:// core.ac.uk/download/pdf/11052852.pdf

42. Montañez Orozco, I., Vargas Sarmiento, C., Cabezas Gutiérrez, M. \& Cuervo Andrade, J. (2010). Colonización micorrizica en plantas de aguacate (Persea americana L.). Revista U.D.C.A Act. \& Div. Cient. 13 (2): 5160. Recuperado de http://www.scielo.org.co/pdf/rudca/ v13n2/v13n2a07.pdf

43. Pellegrino, E. \& Bedini, S. (2014). Enhancing ecosystem services in sustainable agriculture: biofertilization and biofortification of chickpea (Cicer arietinum L.) by arbuscular mycorrhizal fungi. Soil Biol. Biochem. 68, 429-439. 10.1016/j.soilbio.2013.09.030

44. Pérez Melchor, E.J. (2013). Efecto de la micorrización en plantas de aguacate (Persea americana Mill.) variedad Hass y Nativo Antillano durante la fase de vivero. (tesis de pregrado) Ingeniera Agrónoma, Universidad de Caldas Facultad de Ciencias Agropecuarias, Manizales, Colombia. Recuperado de http:// biblio.ucaldas.edu.co:8000/cgi-bin/koha/opac-detail. pl?biblionumber $=46399$

45. Reyes Alemán, J. C. Ferrera Cerrato, R., Cortés Flores, J.L. \& Alarcón, A. (2000). Simbiosis micorrízica y vermicomposta en el desarrollo de porta injertos de aguacate crecidos en sustratos agrícola y forestal. Colegio de Postgraduados, Montecillo, México. Recuperado de http://www.avocadosource.com/Journals/CICTAMEX/CICTAMEX_1998-2001/CICTAMEX_1998-2001_ PG_064-079.pdf

46. Reyes Alemán. J. C., Ferrera Cerrato, R. \& Alarcón, A. (2001). Aplicación de vermicomposta y hongos micorrízicos en la producción de planta de aguacate en vivero. Fundación Salvador Sánchez Colín, CICTAMEX
S.C y Área de Microbiología, Especialidad de Edafología. IRENAT-CP. México. Recuperado de: http:// www.avocadosource.com/journals/cictamex/cictamex_1998-2001/cictamex_1998-2001_pg_080-087.pdf

47. Rivera Espinosa, R.A., Martin Cárdenas, J.V., Calderón Puig, A. \& Torres Hernández, A. (2011). Utilización de cepas eficientes de hongos micorrízicos arbusculares en el desarrollo de portainjertos de aguacate en un sustrato suelo-cachaza. Cultivos tropicales vol.32 no.2, abr.jun, 23.29. http://scielo.sld.cu/scielo.php?script=sci_ar ttext\&pid=S0258-59362011000200007

48. Rivera Páez, F.A., González Salazar, V., González Acosta, J.G. \& Ossa López, P.A. (2016). Caracterización molecular, análisis morfológico y colonización micorrízica en la rizósfera del aguacate (Persea americana Miller) en Caldas, Colombia. Acta Agronómica, vol.65 no.4, 398-405. Versión impresa ISSN 01873180. Recuperado de: https://dx.doi.org/10.15446/ acag.v65n4.51714

49. Sánchez, M. (1999). Endomicorrizas en agroecosistemas colombianos. 1era ed. Palmira, Colombia: Universidad Nacional de Colombia, 1-227.

50. Sieh, D., Watanabe, M., Devers, E. A., Brueckner, F., Hoefgen, R., \& Krajinski, F. (2013). The arbuscular mycorrhizal symbiosis influences sulfur starvation responses of Medicago truncatula. New Phytol. 197, 606-616. doi: 10.1111/nph.12034

51. Sieverding, E. (1991), Vesicular arbuscular mycorrhyza management in tropical agosytems. Plant Physiology, 13, 220-350.

52. Smith S. E. \& Read, D.J. (2008). Mycorrhizal Symbiosis, Third Edition. Elsevier Ltd., London, England.

53. Thies, J. \& Grossman, J.M. (2006). The soil habitat and soil ecology. pp. 59-78. In: Uphoff N. (ed.). Biological approaches to sustainable soil systems. CRC Press, Boca Raton, FL. 764 p.

54. van der Heijden, M. G. A., Martin, F. M., Selosse, M.-A. \& Sanders, I. R. (2015), Mycorrhizal ecology and evolution: the past, the present, and the future. New Phytol, 205: 1406-1423. doi:10.1111/nph.13288

55. Vélez Zabala, F. \& Sánchez de Prager, M. (2014). Dinámica de los hongos de Micorriza Arbuscular (MA) en un Humic Dystrudepts sembrado con maíz Zea mays L. y Abonos Verdes (AV). Revista de Investigación Agraria y Ambiental, 5(1), enero-junio, 69-79. Recuperado de: http://hemeroteca.unad.edu.co/index.php/ riaa/article/view/915/905

56. Whiley, A.W., Chapman, K.R. \& Saranah, J.B. (1988a). Water loss by floral structures of avocado (Persea americana Mill.) cv. Fuerte during flowering. Australian Journal of Agricultural Research, 39, 457-467.

Conflicto de Intereses

Los autores declaran no tener ningún conflicto de intereses

Recibido: agosto 05 de 2016 Aceptado: agosto 30 de 2016 
